Bull. Austral. Math. Soc.

Vol. 49 (1994) [85-90]

\title{
SKEW FIELDS WITH A NON-TRTVIAL GENERALISED POWER CENTRAL RATIONAL IDENTITY
}

\section{Katsuo Chiba}

Let $D$ be a skew field with uncountable centre $K$. The main result in the present paper is as follows: If $D$ satisfies a non-trivial generalised power central rational identity, then $D$ is finite dimensional over $K$. As a corollary we obtain the following result. Let $a$ be an element of $D$ such that $\left(a^{-1} x^{-1} a x\right)^{q(x)} \in K$ for all $x \in D \backslash\{0\}$ where $q(x)$ is a positive integer depending on $x$. Then $a \in K$.

Several authors $[7,8,10]$ have studied skew fields with a certain power central rational identity. In this paper we shall study skew fields with uncountable centre which satisfy a general power central rational identity.

Let $D$ be a skew field with centre $K$ and $K\langle X\rangle$ the free $K$-algebra on a finite set $X=\left\{x_{1}, x_{2}, \ldots, x_{n}\right\}$. We denote by $D\langle X\rangle=D *_{K} K\langle X\rangle$ the free product of $D$ and $K\langle X\rangle$ over $K$ and by $D(X)$ the universal skew field of fractions of $D\langle X\rangle$. Let $d=\left(d_{i}\right)$ be an element of $D^{n}$ and $\alpha_{d}: D\langle X\rangle \rightarrow D$ the $D$-ring homomorphism defined by $\alpha_{d}\left(x_{i}\right)=d_{i}, i=1,2, \ldots, n$. We denote by $\Sigma_{d}$ the set of all matrices over $D\langle X\rangle$ which are mapped by $\alpha_{d}$ to invertible matrices over $D$. Let $\Sigma_{d}^{-1}$ be the set of all entries of inverses $A^{-1}$ over $D(X)$ for all $A \in \Sigma_{d}$. Then $\Sigma_{d}^{-1}$ is a ring and it contains $D\langle X\rangle$ as a subring. Moreover, there is a $D$-ring homomorphism $\beta_{d}: \Sigma_{d}^{-1} \rightarrow D$ which extends $\alpha_{d}$ and satisfies that any element of $\Sigma_{d}^{-1}$ not in the kernel of $\beta_{d}$ has an inverse in $\Sigma_{d}^{-1}$ (see [4, Chapter 7]). Let $f=f\left(x_{i}\right)$ be an element of $D(X)$. If $f$ belongs to $\Sigma_{d}^{-1}$, we say $f$ is defined at $\left(d_{i}\right)$ and write $f\left(d_{i}\right)$ instead of $\beta_{d}(f)$. We say $D$ satisfies a generalised power central rational identity (abbreviated GPCRI) if there is an element $f$ in $D(X)$ satisfying the following condition: if $f$ is defined at $\left(d_{i}\right) \in D^{n}$ then $f\left(d_{i}\right)^{q} \in K$ for some positive integer $q$ which depends only on $\left(d_{i}\right)$. Furthermore, if $f^{p} \notin K$ for any positive integer $p$, we say $D$ satisfies a non-trivial GPCRI $f$.

The purpose of this paper is to prove the following theorem.

Theorem 1. Let $D$ be a skew field with uncountable centre $K$. If $D$ satisfies a non-trivial GPCRI, then $D$ is finite dimensional over $K$.

In [7] Herstein conjectured that any element $a$ of $D$ which satisfies $\left(a^{-1} x^{-1} a x\right)^{q(x)} \in$ $K$ for all $x \in D \backslash\{0\}$ where $q(x)$ depends on $x$ must be central and in [8, p.489] he

Received 16 February 1993

Copyright Clearance Centre, Inc. Serial-fee code: 0004-9729/94 SA2.00+0.00. 
settled the conjecture for the case in which $K$ is an uncountable field of characteristic 0 . As a corollary of Theorem 1 , we settle this conjecture here for the case in which $K$ is an uncountable field of arbitrary characteristic.

Corollary 2. Let $D$ be a skew field with uncountable centre $K$. Let a be an element of $D$ such that $\left(a^{-1} x^{-1} a x\right)^{q(x)} \in K$ for all $x \in D \backslash\{0\}$ where $q(x)$ is a positive integer depending on $x$. Then $a \in K$.

Furthermore, by Theorem 1, we obtain [10, Theorem].

Corollary 3. Let $D$ be a skew field with uncountable centre $K$. Suppose that there is a non-trivial word $w$ in a free group such that every value of $w$ over $D$ is periodic over $K$. Then $D$ is commutative.

Notations and Terminology. Let $E$ be a skew field which contains $D$ and the centre of $E$ contains $K$, the centre of $D$. Let $\left(e_{i}\right) \in E^{n}$ and $f=f\left(x_{i}\right) \in D(X)$. In the same fashion as we previously defined, we say $f$ is defined at $\left(e_{i}\right)$, we use an expression $f\left(e_{i}\right)$ and we say $E$ satisfies a GPCRI $f$. Let $k$ be a field, $R$ a $k$-algebra and $R *_{k} k\langle X\rangle$ the free product of $R$ and $k\langle X\rangle$ over $k$, where $k\langle X\rangle$ is a free algebra on a finite set $X=\left\{x_{1}, x_{2}, \ldots, x_{n}\right\}$. Let $f \in R *_{k} k\langle X\rangle$. We say that $f$ is a generalised power central identity (abbreviated GPCI) of $R$ if for each $\left(r_{i}\right) \in R^{n}$, there exists a positive integer $q$, which depends on $\left(r_{i}\right)$, such that $f\left(r_{i}\right)^{q}$ is a central element of $R$. We shall denote by $D\langle X\rangle[t]$ the polynomial ring over $D\langle X\rangle$ in a central indeterminate $t$ and by $D(X)(t)$ (respectively $D(t)$ ) the quotient skew field of the polynomial ring $D(X)[t]$ (respectively $D[t]$ ). The Laurent series skew field over $D(X)$ (respectively $D$ ) is denoted by $D(X)((t))$ (respectively $D((t))$ ). There is a natural embedding of $D(X)(t)$ (respectively $D(t)$ ) into $D(X)((t))$ (respectively $D((t))$ ), so we shall think of $D(X)(t)$ (respectively $D(t)$ ) as a subring of $D(X)((t)$ ) (respectively $D((t))$ ). If $R$ is a semifir, we denote by $U(R)$ the universal skew field of fractions of $R$.

To prove Theorem 1 , we need several lemmas. We begin with the following.

LEMMA 4. Let $D$ be a skew field with uncountable centre $K$ and $D\left(t_{1}, t_{2}, \ldots, t_{m}\right)$ be a quotient skew field of the polynomial ring $D\left[t_{1}, t_{2}, \ldots, t_{m}\right]$, where $t_{i}, i=$ $1,2, \ldots, m$, are central indeterminates. If $D$ satisfies a GPCRI $f=f\left(x_{i}\right) \in D(X)$ then $D\left(t_{1}, t_{2}, \ldots, t_{m}\right)$ also satisfies the GPCRI $f$.

Proof: We first show that $D\left(t_{1}\right)$ satisfies the GPCRI $f$. Suppose $f=f\left(x_{i}\right)$ is defined at $\left(h_{i}\left(t_{1}\right)\right) \in D\left(t_{1}\right)^{n}$. Since $K$ is uncountable, $f$ is defined at $\left(h_{i}(u)\right) \in D^{n}$ for uncountably many elements $u \in K$. Then, by the Pigeon-Hole Principle we can find a positive integer $q$ such that $f\left(h_{i}(u)\right)^{q} \in K$ for infinitely many elements $u \in K$. By [10, Lemma 1], $f\left(h_{i}\left(t_{1}\right)\right)^{q}$ is central in $D\left(t_{1}\right)$. Thus $D\left(t_{1}\right)$ satisfies the GPCRI $f$. Since $D\left(t_{1}, t_{2}, \ldots, t_{i+1}\right)=D\left(t_{1}, t_{2}, \ldots, t_{i}\right)\left(t_{i+1}\right)$, by induction on $m$ it follows that $D\left(t_{1}, t_{2}, \ldots, t_{m}\right)$ satisfies the GPCRI $f$. This completes the proof. 
LEMma 5. Let $D$ be a skew field with uncountable centre $K$ and $L$ a field containing $K$. Suppose $g=g\left(x_{i}\right) \in D *_{K} K\langle X\rangle=D\langle X\rangle$ is a GPCI of $D$. Then $g$ is also a GPCI of $D \otimes_{K} L$.

Proof: Let $a_{i} \in D \otimes_{K} L, i=1,2, \ldots, n$. Then there are a polynomial ring $D\left[t_{1}, t_{2}, \ldots, t_{m}\right], n$ elements $h_{i} \in D\left[t_{1}, t_{2}, \ldots, t_{m}\right], i=1,2, \ldots, n$, and a $D$-ring homomorphism $\phi: D\left[t_{1}, t_{2}, \ldots, t_{m}\right] \rightarrow D \otimes_{K} L$ such that $\phi\left(t_{j}\right) \in L, j=1,2, \ldots, m$, and $\phi\left(h_{i}\right)=a_{i}, i=1,2, \ldots, n$. It is clear that $g$ is a GPCRI of $D$. Hence, by Lemma $4, D\left(t_{1}, t_{2}, \ldots, t_{m}\right)$ satisfies the GPCRI $g$. Therefore we can find a positive integer $q$ such that $g\left(h_{i}\right)^{q} \in K\left[t_{1}, t_{2}, \ldots, t_{m}\right]$, the centre of $D\left[t_{1}, t_{2}, \ldots, t_{m}\right]$, and hence $g\left(a_{i}\right)^{q}=\phi\left(g\left(h_{i}\right)^{q}\right) \in L$. Thus $D \otimes_{K} L$ satisfies the GPCI $g$.

LEMMA 6. Let $D$ be a skew field with uncountable centre $K$. If $D$ satisfies a GPCI in $D\langle X\rangle \backslash D$, then $D$ is finite dimentional over $K$.

Proof: Let $g=g\left(x_{i}\right) \in D\langle X\rangle \backslash D$ be a GPCI of $D$, let the $X$-degree of $g$ be $m$, and let $D\langle X\rangle[t]$ be the polynomial ring over $D\langle X\rangle$ in a central indeterminate $t$. Then we can express $g\left(x_{i} t\right) \in D\langle X\rangle[t]$ in the form

$$
g\left(x_{i} t\right)=g_{m}\left(x_{i}\right) t^{m}+g_{m-1}\left(x_{i}\right) t^{m-1}+\ldots+g_{0}
$$

where $g_{j}\left(x_{i}\right) \in D\langle X\rangle$ is homogeneous and of $X$-degree $j$ for $j=0,1,2, \ldots, m$. Let $\left(d_{i}\right) \in D^{n}$. Since $K$ is uncountable, by the Pigeon-Hole Principle, we can find a positive integer $q$ such that $g\left(d_{i} u\right)^{q} \in K$ for infinitely many elements $u \in K$. By a van der Monde determinant argument, we have that $g_{m}\left(d_{i}\right)^{q} \in K$. Thus we may assume that $g$ is homogeneous. Let us write $g$ in the following form:

$$
g=\Sigma_{i} e_{i} x_{i 1} d_{i 1} x_{i 2} d_{i 2} \ldots x_{i m} d_{i m}
$$

where $x_{i j} \in\left\{x_{1}, x_{2}, \ldots, x_{n}\right\},\left\{e_{i}, d_{i j}\right\} \subset D$ and $e_{i} \neq 0$. Let us denote the elements $d_{i j}$ by $d_{1}, d_{2}, \ldots, d_{h}$. We may assume that $d_{1}, d_{2}, \ldots, d_{h}$ are $K$-linearly independent. Now, let $L$ be a maximal commutative subfield of $D$. Then $R=D \otimes_{K} L$ is a dense ring of linear transformations on $D$ considered as a right vector space over $L$. Assume $[D: K]=\infty$. Then, by $\left[1\right.$, Corollary $\left.8^{*}\right], D \otimes_{K} L$ has no finite ranked transformation. By [1, Lemma 11] we obtain $m+1$ elements $v_{0}, v_{1}, v_{2}, \ldots, v_{m}$ in $D$ such that the elements of $V=\left\{d_{i} v_{j}: i=1,2, \ldots, h, j=0,1,2, \ldots, m\right\}$ are right $L$-linearly independent. Consider the finite set of the $x$ 's which appear in the monomial $e_{1} x_{11} d_{11} x_{12} d_{12} \ldots x_{1 m} d_{1 m}$. Without loss of generality we may assume that these are $x_{1}, x_{2}, \ldots, x_{u}$. Since $R=D \otimes_{K} L$ acts densely on $D$, we can find $u$ elements $c_{k}$ $k=1,2, \ldots, u$, in $R$ which act on $V$ such that

(1) $c_{k} d_{11} v_{1}=e_{1}^{-1} v_{m}$ if $x_{11}=x_{k}$,

(2) $c_{k} d_{1 j} v_{j}=v_{j-1}$ if $x_{1 j}=x_{k}$ for $j=2, \ldots, m$,

(3) $c_{k} d_{\lambda} v_{\mu}=0 \quad$ otherwise. 
Let $c=g\left(c_{1}, c_{2}, \ldots, c_{u}, 0, \ldots, 0\right)$. Then we have $c v_{m}=v_{m}$. By Lemma $5, g$ is a GPCI of $R$. Hence there is a positive integer $s$ such that $c^{*}=1$. On the other hand, by the definitions of $c_{k}, k=1,2, \ldots, u$, it follows that $c v_{0}=0$, a contradiction. Thus $[D: K]<\infty$. This proves the lemma.

Lemma 7. If $f=f\left(x_{i}\right) \in D(X) \backslash D$ is defined at $\left(d_{i}\right) \in D^{n}$, then $f$ is defined at $\left(d_{i}+x_{i} t\right) \in D(X)((t))^{n}$ and $f\left(d_{i}+x_{i} t\right)$ has the representation:

$$
f\left(d_{i}+x_{i} t\right)=f_{0}+f_{1} t+f_{2} t^{2}+\ldots
$$

where $f_{0}=f\left(d_{i}\right), f_{i} \in D\langle X\rangle$ is homogeneous with $X$-degree $i$ for $i \geqq 1$. Moreover, there exists $i \geqq 1$ such that $f_{i} \neq 0$.

Proof: It is easy to show that $f$ is defined at $\left(d_{i}+x_{i} t\right) \in D(X)((t))^{n}$ and $f\left(d_{i}+x_{i} t\right)$ has the above representation. We show that $f_{i} \neq 0$ for some $i \geqq 1$. Let

$$
R_{1}=\left\{q(t) p(t)^{-1} \in D(X)(t) ; p(t), q(t) \in D(X)[t] \text { and } p(1) \text { invertible in } D(X)\right\}
$$

Then, by [2, Lemma 5], $R_{1}$ is a subring of $D(X)(t)$ and there is a ring homomorphism $\phi: R_{1} \rightarrow D(X)$ such that $\phi\left(q(t) p(t)^{-1}\right)=q(1) p(1)^{-1}$. Clearly we have $f\left(d_{i}+x_{i} t\right) \epsilon$ $R_{1}$. Let $\psi: D(X) \rightarrow D(X)$ be the $D$-automorphism defined by $\psi\left(x_{i}\right)=x_{i}-d_{1}$, $i=1,2, \ldots, n$. Suppose $f\left(d_{i}+x_{i} t\right)=f_{0} \in D$. Then we have $\psi \phi\left(f\left(d_{i}+x_{i} t\right)\right)=$ $f\left(x_{i}\right)=f_{0} \in D$, a contradiction. This proves the lemma.

We are now ready to prove Theorem 1.

Proof of Theorem 1: Assume to the contrary that $[D: K]=\infty$. Let $f=$ $f\left(x_{i}\right) \in D(X) \backslash D$ be a non-trivial GPCRI of $D$. Then by [5, Theorem 7.2.7] we can find an element $\left(d_{i}\right) \in D^{n}$ such that $f$ is defined at $\left(d_{i}\right)$ and $f\left(d_{i}\right) \neq 0$. Since $f$ is a GPCRI of $D$, there is a positive integer $p$ such that $f\left(d_{i}\right)^{p} \in K$. We show $f\left(x_{i}\right)^{p} \notin D$. Suppose $f\left(x_{i}\right)^{p} \in D$. Since $f\left(x_{i}\right)^{p}$ is defined at $\left(d_{i}\right)$, it follows that $f\left(x_{i}\right)^{p}=f\left(d_{i}\right)^{p} \in K$, contradicting the fact that $f\left(x_{i}\right)$ is a non-trivial GPCRI of $D$. Hence, by Lemma 7 we have the representation in $D(X)((t))$ :

$$
f\left(d_{i}+x_{i} t\right)^{p}=f_{0}+f_{m} t^{m}+f_{m+1} t^{m+1}+\ldots
$$

where $f_{0}=f\left(d_{i}\right)^{p} \neq 0,0 \neq f_{m}=f_{m}\left(x_{i}\right) \in D\langle X\rangle$ with $f_{m}$ homogeneous of $X$-degree $m$. It is easy to see that $f\left(x_{i}\right)$ is defined at $\left(d_{i}+e_{i} t\right) \in D(t)^{n}$ for any $\left(e_{i}\right) \in D^{n}$. By Lemma $4 D(t)$ satisfies the GPCRI $f$, so for each $\left(e_{i}\right) \in D^{n}$ we can find an integer $r$ such that $f\left(d_{i}+e_{i} t\right)^{p r} \in K$. If the characteristic of $D$ is zero, then the first two terms in $f\left(d_{i}+e_{i} t\right)^{p r}=\left\{f_{0}+f_{m}\left(e_{i}\right) t^{m}+\ldots\right\}^{r}$ are $f_{0}^{r}+r f_{0}^{r-1} f_{m}\left(e_{i}\right) t^{m}$, 
so that $f_{m}\left(e_{i}\right)$ must be central. If the characteristic of $D$ is $\kappa \neq 0$, then we write $r=k N$ where $k$ is a power of $\kappa$ and $N$ is prime to $\kappa$. Then the first two terms in $f\left(d_{i}+e_{i} t\right)^{p r}=\left\{f_{0}+f_{m}\left(e_{i}\right) t^{m}+\ldots\right\}^{r}$ are $f_{0}^{r}+N f_{0}^{r-k} f_{m}\left(e_{i}\right)^{k} t^{m k}$ so that $f_{m}\left(e_{i}\right)^{k}$ is central. Thus $f_{m}\left(x_{i}\right)$ is a GPCI of $D$. By Lemma $6, D$ is finite dimensional over $K$, a contradiction. This completes the proof.

For the proof of Corollaries 2 and 3 , we recall

LEMMA 8. Let $F$ be a free group on the set $X=\left\{x_{1}, x_{2}, \ldots, x_{n}\right\}$ and $K[F]$ the group algebra over $K$. Then there is a natural isomorphism $D(X)=U\left(D *_{K} K\langle X\rangle\right) \simeq$ $U\left(D *_{K} K[F]\right)$.

Proof: Let $K(X)=U(K\langle X\rangle)$ and $K(F)=U(D[F])$. Then, by [9, Theorem 2] we have a natural isomorphism $K(X) \simeq K(F)$. By [5, Lemma 5.4.1 (ii)], we have natural isomorphisms $U\left(D *_{K} K\langle X\rangle\right) \simeq U\left(D *_{K} K(X)\right)$ and $U\left(D *_{K} K[F]\right) \simeq$ $U\left(D *_{K} K(F)\right)$. Thus we have a natural isomorphism $U\left(D *_{K} K\langle X\rangle\right) \simeq$ $U\left(D *_{K} K[F]\right)$.

Proof of Corollary 2: Assume $a \notin K$. Then also $a^{-1} \notin K$. By Lemma 8 and [3, Corollary 8.1] $a^{-1} x_{1}^{-1} a x_{1}$ is a non-trivial GPCRI of $D$. Then $[D: K]<\infty$ by Theorem 1. Hence $[D(t): K(t)]<\infty$, where $K(t)$ is the centre of $D(t)$, as is well known. By Lemma $4, D(t)$ satisfies the GPCRI $a^{-1} x_{1}^{-1} a x_{1}$, and hence, by [7, Sublemma] for each $d \in D$ we can find a positive integer $q$, which depends on $d$, such that $\left\{a^{-1}(1+d t)^{-1} a(1+d t)\right\}^{q}=\left\{1+\left(d-a^{-1} d a\right) t+\ldots\right\}^{q}=1$. By the same argument as in the proof of Theorem 1, we obtain a positive integer $N$ such that $\left(d-a^{-1} d a\right)^{N}=0$. Hence $d-a^{-1} d a=0$. Therefore we have $a \in K$, a contradiction. This completes the proof.

Proof of Corollary 3: Let $\boldsymbol{w}$ be a non-trivial word in a free group of rank $n$ such that every value of $w$ over $D$ is periodic over $K$. By Lemma $8, w$ is a non-trivial GPCRI of $D$. Then, by Theorem $1[D: K]<\infty$. Suppose $D$ is not commutative. Then, by [6, Theorem 2.1] $D \backslash\{0\}$ contains a free subgroup $G$ of rank two. As is wellknown, $G$ contains a free subgroup of rank $n$, which is a contradiction. This completes the proof.

\section{REFERENCES}

[1] S.A. Amisur, 'Generalized polynomial identities and pivotal monomials', Trans. Amer. Math. Soc. 114 (1965), 210-226.

[2] S.A. Amisur, 'Rational identities and applications to algebra and geometry', J. Algebra 3 (1966), 304-359.

[3] G.M. Bergman, 'The diamond lemma for ring theory', Adv. in Math. 29 (1978), 178-218.

[4] P.M. Cohn, Free rings and their relations (Academic Press, New York and London, 1971). 
[5] P.M. Cohn, Skew field constructions, London Math. Soc. Lecture Notes No. 27 (Cambridge University Press, Cambridge, 1977).

[6] J.Z. Goncalves, 'Free groups in subnormal subgroups and the residual nilpotence of the groups of units of groups rings', Canad. Math. Bull. 27 (1984), 365-370.

[7] I.N. Herstein, 'Multiplicative commutators in division rings', Israel J. Math. 31 (1978), 180-188.

[8] I.N. Hetstein, 'Multicative commutators in division rings II', Rend. Circ. Mat. Palermo II 29 (1980), 485-489.

[9] J. Lewin, 'Fields of fractions for group algebras of free groups', Trans. Amer. Math. Soc. 192 (1974), 339-346.

[10] L. Makar-Limanov and P. Malcolmson, 'Words periodic over the center of a division ring', Proc. Amer. Math. Soc. 93 (1985), 590-592.

Niihama National College of Technology

7 - 1 Yagumo-Cho

Niihama 792

Japan 